\title{
Million RNA Copies per Milliliter
}

National Cancer Institute

\section{Source}

National Cancer Institute. Million RNA Copies per Milliliter. NCI Thesaurus. Code C98760.

A unit of measurement equal to one million ribonucleic acid (RNA) copies per unit of volume equal to one milliliter. 